\title{
Video Article \\ Stem Cell Transplantation Strategies for the Restoration of Cognitive Dysfunction Caused by Cranial Radiotherapy
}

\author{
Munjal M. Acharya* ${ }^{1}$, Dante E. Roa* ${ }^{1}$, Omar Bosch ${ }^{1}$, Mary L. Lan* ${ }^{1}$, Charles L. Limoli ${ }^{1}$ \\ ${ }^{1}$ Department of Radiation Oncology, University of California Irvine \\ * These authors contributed equally
}

Correspondence to: Charles L. Limoli at climoli@uci.edu

URL: https://www.jove.com/video/3107

DOI: doi:10.3791/3107

Keywords: Medicine, Issue 56, neuroscience, radiotherapy, cognitive dysfunction, stem cells, transplantation

Date Published: 10/18/2011

Citation: Acharya, M.M., Roa, D.E., Bosch, O., Lan, M.L., Limoli, C.L. Stem Cell Transplantation Strategies for the Restoration of Cognitive Dysfunction Caused by Cranial Radiotherapy. J. Vis. Exp. (56), e3107, doi:10.3791/3107 (2011).

\section{Abstract}

Radiotherapy often provides the only clinical recourse for those afflicted with primary or metastatic brain tumors. While beneficial, cranial irradiation can induce a progressive and debilitating decline in cognition that may, in part, be caused by the depletion of neural stem cells. Given the increased survival of patients diagnosed with brain cancer, quality of life in terms of cognitive health has become an increasing concern, especially in the absence of any satisfactory long-term treatments.

To address this serious health concern we have used stem cell replacement as a strategy to combat radiation-induced cognitive decline. Our model utilizes athymic nude rats subjected to cranial irradiation. The ionizing radiation is delivered as either whole brain or as a highly focused beam to the hippocampus via linear accelerator (LINAC) based stereotaxic radiosurgery. Two days following irradiation, human neural stem cells (hNSCs) were stereotaxically transplanted into the hippocampus. Rats were then assessed for changes in cognition, grafted cell survival and for the expression of differentiation-specific markers 1 and 4-months after irradiation. Our cognitive testing paradigms have demonstrated that animals engrafted with hNSCs exhibit significant improvements in cognitive function. Unbiased stereology reveals significant survival (10-40\%) of the engrafted cells at 1 and 4-months after transplantation, dependent on the amount and type of cells grafted. Engrafted cells migrate extensively, differentiate along glial and neuronal lineages, and express a range of immature and mature phenotypic markers.

Our data demonstrate direct cognitive benefits derived from engrafted human stem cells, suggesting that this procedure may one day afford a promising strategy for the long-term functional restoration of cognition in individuals subjected to cranial radiotherapy. To promote the dissemination of the critical procedures necessary to replicate and extend our studies, we have provided written and visual documentation of several key steps in our experimental plan, with an emphasis on stereotaxic radiosurgey and transplantation.

\section{Video Link}

The video component of this article can be found at https://www.jove.com/video/3107/

\section{Protocol}

Our experimental plan is schematically diagrammed in Figure 1.

\section{Growth and preparation of human neural stem cells (NSCs) for transplantation}

1. The EnStem-A cell line (EMD Millipore) was used in this study. NSCs are routinely validated by the manufacturer for high levels of expression of the multipotent markers nestin and Sox2, and low-level expression of the pluripotent marker Oct-4, along with the ability to differentiate into multiple neuronal phenotypes and to maintain a normal karyotype after multiple passages. Under our growth conditions, these NSCs displayed abundant expression of Sox 2 and nestin and retained their ability to differentiate, as described previously ${ }^{1}$. NSCs were grown on poly-L-ornithine $(20 \mu \mathrm{g} / \mathrm{ml})$ and laminin $(5 \mu \mathrm{g} / \mathrm{ml})$ coated tissue culture treated flasks. Cells were grown in neural expansion medium (Millipore) supplemented with L-glutamine $(2 \mathrm{mM})$ and basic fibroblast growth factor (bFGF; $20 \mathrm{ng} / \mathrm{ml}$ ). Adherent monolayers of NSCs were passaged every other day (1:2) with accutase as the dissociation agent ${ }^{1}$. For transplantation studies, NSCs were used at passages below 10.

2. NSCs were labeled with 5-bromo-2'-deoxyuridine (BrdU) by supplementing the growth media with $4 \mu \mathrm{M}$ BrdU for three days prior to transplantation. To verify the BrdU labeling index (i.e. percentage of BrdU positive cells), cells were plated on chamber slides and processed for BrdU detection using standard immunocytochemical approaches. Cells utilized for transplantation routinely exhibit labeling indices in excess of $90 \%{ }^{2}$. Alternatively, transplanted human stem cells were detected using the human specific nuclear antigen marker (HuNu) ${ }^{2}$.

3. On the day of transplantation, accutase and neural expansion media were pre-warmed in a $37^{\circ} \mathrm{C}$ water bath. Cells were placed in incubation medium (neural expansion media containing $10 \mu \mathrm{M}$ of Y-27632) for one hour. Y-27632 (ROCK inhibitor, EMD-Calbiochem) was used to improve the survival of NSCs post-transplantation. 
4. After one hour, incubation media was removed and cells were treated with accutase for 5 minutes. After this brief treatment add an equal volume of neural expansion media to neutralize the accutase and strain the cells through a $70 \mu \mathrm{M}$ cell strainer.

5. Count the cells with a haemocytometer and prepare $1.0 \times 10^{5}$ live NSCs per microliter in injection medium (neural expansion media containing $10 \mu \mathrm{M}$ of $\mathrm{Y}-27632,40 \mathrm{ng} / \mathrm{ml} \mathrm{bFGF}, 20 \mathrm{ng} / \mathrm{ml} \mathrm{BDNF}$ ).

6. Cells were stored in neural expansion media (as described in 1.5) and kept on ice until the time of transplantation, and should be used within $6 \mathrm{~h}$ to minimize cell death by prolonged cold-storage.

\section{Radiotherapy - treatment planning and irradiation}

1. Sedated athymic nude rat(s) were placed in a laboratory MRI scanner in the prone position with the skull at the superior end (head first). This 3 tesla scanner is designed for small animal scans. It can provide exquisite soft-tissue contrast with high spatial resolution cross-sectional (axial) images. The scan focused on the skull region and a set of 22 T2 E60 weighted images with 0.8 mm image thickness were generated. These images provide necessary information to identify the left and right hippocampi within the rat's brain.

2. Following the MRI scan (24h), animals were sedated [anesthesia cocktail (ketamine, $30 \mathrm{mg} / \mathrm{kg}$, xylazine, $2.5 \mathrm{mg} / \mathrm{kg}$ and acepromazine, $1 \mathrm{mg} /$ $\mathrm{kg}$ )] and placed in a radiation oncology CT scanner where another cross-sectional (axial) image volume was generated. The rat was placed in the same or close to the same position as used for the MRI scan and the CT unit was set to scan the skull region. A study of $106 \mathrm{CT}$ images with $0.8 \mathrm{~mm}$ image thickness was generated which became the CT treatment planning data.

3. Treatment Planning

a. The MRI and CT image data were transferred to the ECLIPSE (Varian Medical Systems, Palo Alto, CA) treatment planning software via DICOM (Digital-Imaging and Communications in Medicine). ECLIPSE is a sophisticated and commercially available software used in radiation oncology to model and design the best radiation treatment plan for a patient. It incorporates organ density information that is extracted from the CT image data and used to calculate the radiation dose distribution within the body.

b. Cover the animal using a small surgical drape with only the head exposed. Position the animal head firmly within the stereotaxic frame (Benchmark Digital, Leica-myNeurolab) by inserting ear bars into the external auditory meatus. Take extreme care when sliding the tip of the ear bar into the ear canal. Place the incisor bar by hooking the rat's upper incisors and adjusting the bar height to the standard reference point, then tighten nose clamp.

c. Center the position of the head between the ear bars with minimal lateral movement $( \pm 4 \mathrm{~mm})$ to achieve stereotaxic zero.

d. The next step in the process is to determine the irradiation technique to be used. Intensity-modulated radiation therapy (IMRT) and volumetrically-modulated arc therapy (VMAT) in the form of RapidArc are 2 high-precision irradiation techniques that use a 6 MV photon beam to deliver a radiation dose ${ }^{3-6}$. The difference between these techniques is that IMRT delivers a dose using several static trajectories each corresponding to a different direction but converging at the target volume more suitable for extremely small target volumes. RapidArc on the other hand, delivers a dose dynamically through one or more arcs focused on the target region (Fig. 4).

e. Regardless of delivery technique, information on target dose, target conformity and dose constraints to critical organs have to be entered in the dose optimization window in ECLIPSE. This information along with organ volumes is used to tailor the dose distribution, which is achieved by continually varying the beam attenuation during irradiation.

f. Once a plan was generated, ECLIPSE provides the calculated dose superimposed to the axial, coronal and sagittal images (Fig. 5) as well as in dose-volume histogram (DVH) format (Fig. 6). It is at this point when the plan must be evaluated to determine if it is suitable for delivery or needs to be improved.

4. Irradiation Process

1. Once a plan was approved for delivery, digitally-reconstructed radiograph (DRR) images were generated from the CT treatment planning data in ECLIPSE. These are orthogonal images weighted on bone density to highlight the skull and other bony landmarks. Thereafter, treatment plan and DRRs are sent to the treatment delivery computer system via DICOM.

2. The delivery system controls a Varian Trilogy Linear Accelerator normally used for radiotherapy of humans. The accelerator is equipped with 120 computer controlled thin multileaf collimators (MLC) and a diagnostic quality x-ray imaging system included within the on-board imaging $(\mathrm{OBI})$ system. This delivery system uploads the ECLIPSE-specific information and reconfigures the collimator parameters in the accelerator for delivering the planned dose.

3. At this point the rat was prepared for irradiation, sedated and covered with a paper blanket to keep it warm. After a few minutes, the rat, with its blanket but with the skull exposed, was placed on the treatment table in the same position used to generate the CT scan. This is a critical step because position accuracy is directly correlated to accuracy in dose delivery.

4. Once the rat was positioned properly on the treatment table, a set of orthogonal X-ray images is taken using the Trilogy's OBI system These are high resolution images that were subsequently fused to the ECLIPSE generated DRRs using the OBI image fusion software.

5. The X-ray images and DRRs were digitally matched using software that provide information on table position shifts that must be made to achieve co-registration of the images (Fig. 7). After reviewing this information and verifying that the resultant shifts do not inadvertently lead into any collisions between the accelerator and the treatment table, the computer applies the shifts and the treatment table moves automatically to the desired position.

6. At this point, irradiation commences. To deliver a $10 \mathrm{~Gy}$ dose with a 6-field IMRT plan, the beam-on time is approximately 10 minutes while a two-arc RapidArc takes $\sim 3$ minutes. After treatment delivery is complete, the rat was removed from the treatment room and allowed to recover in a holding cage kept on a heating pad.

\section{Stereotaxic surgery for intra-hippocampal transplantation of human stem cells}

1. Animals husbandry and surgical preparation

a. For this study, we employed two month old ATN rats acquired from National Cancer Institute (strain 0N01 Cr:NIH-rnu). Animals were kept in sterile cages and maintained in a temperature- and light controlled barrier-A environment with 12-h/12-h light/dark cycle. Rats were provided autoclaved food and water ad libitum and care was taken to prevent eye infection by cleaning eyes every week with Vetropolycin eye ointment (Western Medical Supply, Arcadia, CA). 
b. For stereotaxic surgery, rats were anesthetized with an i.p. injection of anesthesia cocktail (ketamine, $30 \mathrm{mg} / \mathrm{kg}, \mathrm{xylazine,} 2.5 \mathrm{mg} / \mathrm{kg}$ and acepromazine, $1 \mathrm{mg} / \mathrm{kg}$ ). Complete anesthesia was induced after 10-15 minutes and sedation was monitored using palpable reflex (toe pinch). If required during surgery, $15-20 \%$ of the original dose may be given to maintain sufficient anesthesia.

c. Remove fur from head using electric clippers. Approximately $200 \%$ of surgery area should be shaved to prevent infection by hairs. Clean shaved part using providone/iodine ( 3 times) followed by $70 \%$ alcohol ( 3 times) before incision.

2. Stereotaxic surgery

a. Stereotaxic instruments (digital monitor and frame), micromotor drill, dry bed sterilizer and controllers should be kept in a Laminar flow hood to prevent possible infection during surgery procedure in ATN rats. A stereotaxic stage with an embedded heating pad can be used to keep the animals warm during surgery. We used 'Kimberly-Clark Safeskin Purple Nitrile Sterile Exam Gloves' thoughout the surgery. These gloves are individually packed in sterile bag (Cat. no. 55093). In addition, surgeon used $70 \%$ alcohol spray to sterilize hands during surgery.

b. Cover the animal using a small surgical drape with only the head exposed. Position the animal head firmly within the stereotaxic frame (Benchmark Digital, Leica-myNeurolab) by inserting ear bars into the external auditory meatus. Take extreme care when sliding the tip of the ear bar into the ear canal. Place the incisor bar by hooking the rat's upper incisors and adjusting the bar height to the standard reference point, then tighten nose clamp.

c. Center the position of the head between the ear bars with minimal lateral movement $( \pm 4 \mathrm{~mm})$ to achieve stereotaxic zero.

d. Following these positioning steps, apply lubricating eye ointment to prevent drying and protect them from possible iodine or alcohol spills. Use this ointment at least 2 times during surgery.

e. Make midline skin incision $(2 \mathrm{~cm})$ along scalp using sterile scalpel, and clean using sterile cotton tipped applicator. Avoid damage in caudal and neck muscles areas.

f. By using dissecting retractor, hold the periosteum open and clear soft tissue using cotton tipped applicator dipped in $1 \%$ hydrogen peroxide (made in PBS). To stop bleeding, maintain firm pressure for at least 1 min using cotton tipped applicator or gauze. Apply firm scraping motion to clean skull and dabbing motion for skin until skull sutures, bregma and lambda were visible.

g. Precise stereotaxic coordinates, referenced to the bregma, were determined using the rat brain atlas ${ }^{7}$. Stem cell transplantation was done at four distinct sites for each hemisphere using the following stereotaxic coordinates:

i. Anterio-posterior (AP) $3.0 \mathrm{~mm}$ from bregma, medio-lateral (ML) $1.8 \mathrm{~mm}$ from midline, and dorso-ventral (DV) $3.2 \mathrm{~mm}$ from the surface of the brain.

ii. AP, $3.6 \mathrm{~mm}$; ML, $2.5 \mathrm{~mm}$; DV, $3.2 \mathrm{~mm}$

iii. AP, $4.2 \mathrm{~mm}$; ML, $3.2 \mathrm{~mm}$; DV, $3.2 \mathrm{~mm}$

iv. $\mathrm{AP}, 4.2 \mathrm{~mm}$; ML, $3.2 \mathrm{~mm}$; DV, $3.2 \mathrm{~mm}$

h. Once the bregma has been identified, all three coordinates (AP, ML and DV) should be zeroed on the digital display/controller (on the digital stereotaxic frame). Then proceed to mark the precise transplantation sites (using the foregoing coordinates) with a fine point marker pen attached to a small probe holder on the frame.

i. Drill a $0.35 \mathrm{~mm}$ hole (no. $1 / 4$ dental burr, carbide vanadium drill bits) through the skull using the foot pedal controlled micromotor drill (Leica-myNeurolab). Take care to prevent damage to the dura membrane. If bleeding occurs during drilling, apply firm pressure using cotton tipped applicator. Do not apply alcohol or iodine at the drill site, as this will cause irritation to the animal.

j. Rats received bilateral intra-hippocampal injections of a suspension of NSCs injected in a maximum volume of $1 \mu \mathrm{L}$ using a $5 \mu \mathrm{L}$ Hamilton microsyringe (30 gauge). The precise number of cells within this volume will vary according to experimental specifics. To accomplish this, the microsyringe was attached to a small probe holder on the stereotaxic frame. Carefully insert the tip of the needle in the skull until reaching the surface of the brain (i.e. meninges). At this point, the DV coordinate should be zeroed on the digital display/ controller, then gently insert the needle to the desired depth (DV, $3.2 \mathrm{~mm}$ ). Wait 1 min before initiating any injection of cells.

k. Inject $0.25 \mu \mathrm{L}$ volume/min (slow release) using a timer. Once a total of $1 \mu \mathrm{L}$ is injected, wait 8 min before retracting needle from transplantation site. After this time, slowly retract the needle from the transplantation site $(0.5 \mathrm{~mm} / \mathrm{min})$ to prevent capillary reflux of the injection contents back through the needle track.

I. Follow steps 3.2(j-k) for the remaining transplantation sites (4 per hemisphere).

$\mathrm{m}$. Remove the skin retractor from the skull and use blunt tweezers to gently pull back retracted skin and apply $4-5$ sterile stainless clips using Autoclips surgical suture applier (Leica-myNeurolab).

$\mathrm{n}$. After removing the animal from the frame, inject with analgesic (Buprenorphin, $0.1 \mathrm{mg} / \mathrm{kg}$, s.c., every $12 \mathrm{~h}$ ) and Lactated Ringer's solution $(5 \mathrm{~mL} / 250 \mathrm{~g}$ adult rat, s.c.).

o. Place the animal back in its cage that should be kept on a heating pad. Monitor the animal until it becomes conscious before returning to its holding room. Place some moistened pellets (animal chow) and transgel in a separate petri dish in each animal cage. Alternatively, DietGel Recovery $\left(\mathrm{ClearH}_{2} \mathrm{O}\right.$, Portland, ME) can be used as a source of water and nutrients.

p. Monitor animals during their recovery time and apply analgesia (buprenorphine $0.02 \mathrm{mg} / \mathrm{kg}$ (every $12 \mathrm{~h}$ for 2 days). Check for signs of pain, distress, reddening or infection of surgery site. Apply providone/iodine or neosporin ointment once daily (till 2-3 days) to prevent possible infection. If any signs of infection, pain or distress persist following analgesic or antibiotic treatment within 12 hours of surgery, consult veterinarian or animal care staff for further assistance. 


\section{Representative results:}

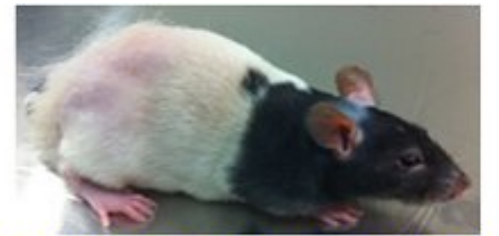

Athymic nude rats (ATN)

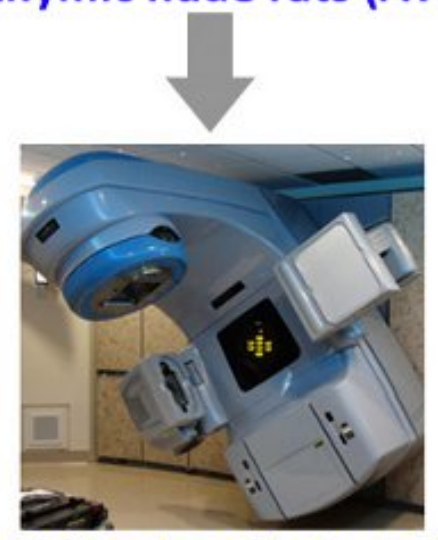

RapidArc irradiation 10Gy

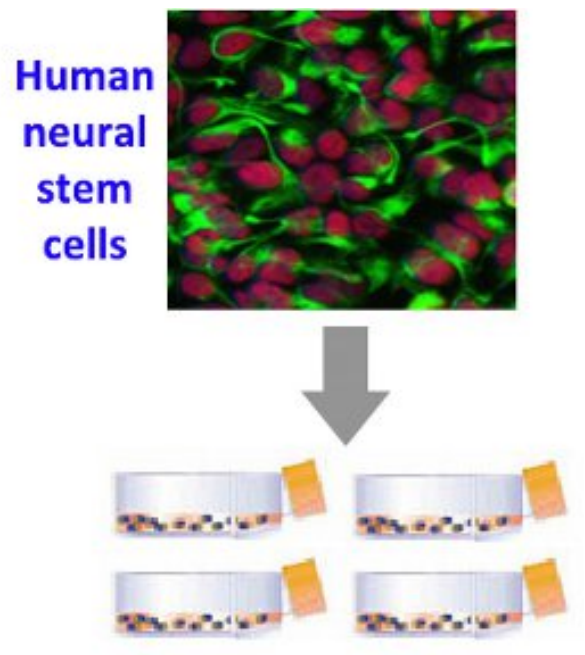

In vitro proliferation, characterization and BrdU labeling

\section{2-days post-irradiation}
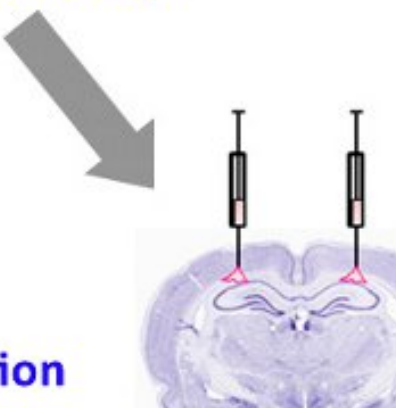

Figure 1. Schematic representation of our experimental plan.

\section{Stem cell grafting or sham surgery (bilateral)}




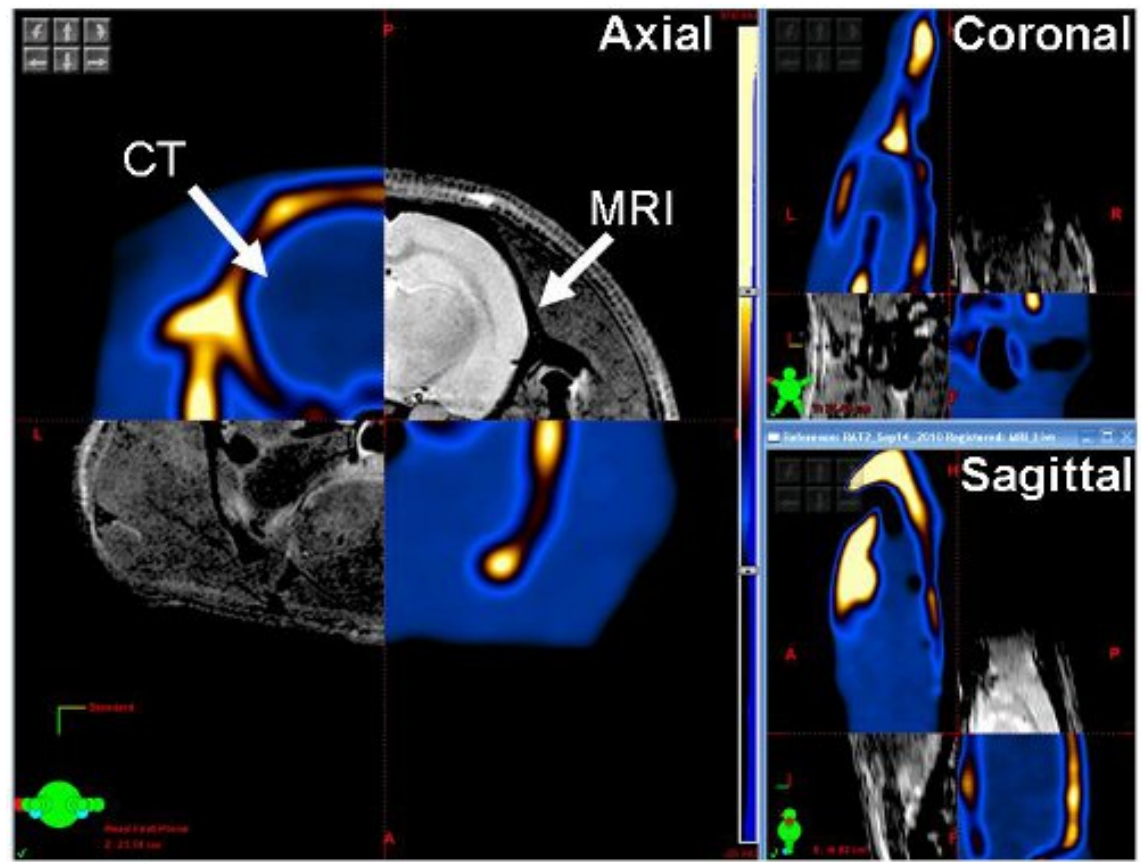

Figure 2. Fusion of CT (bony anatomy) and MRI (soft-tissue) images within the ECLIPSE software. Matching of axial, coronal, and sagittal sections allow for the co-registry of the critical anatomical features of the rat brain derived from each imaging modality.

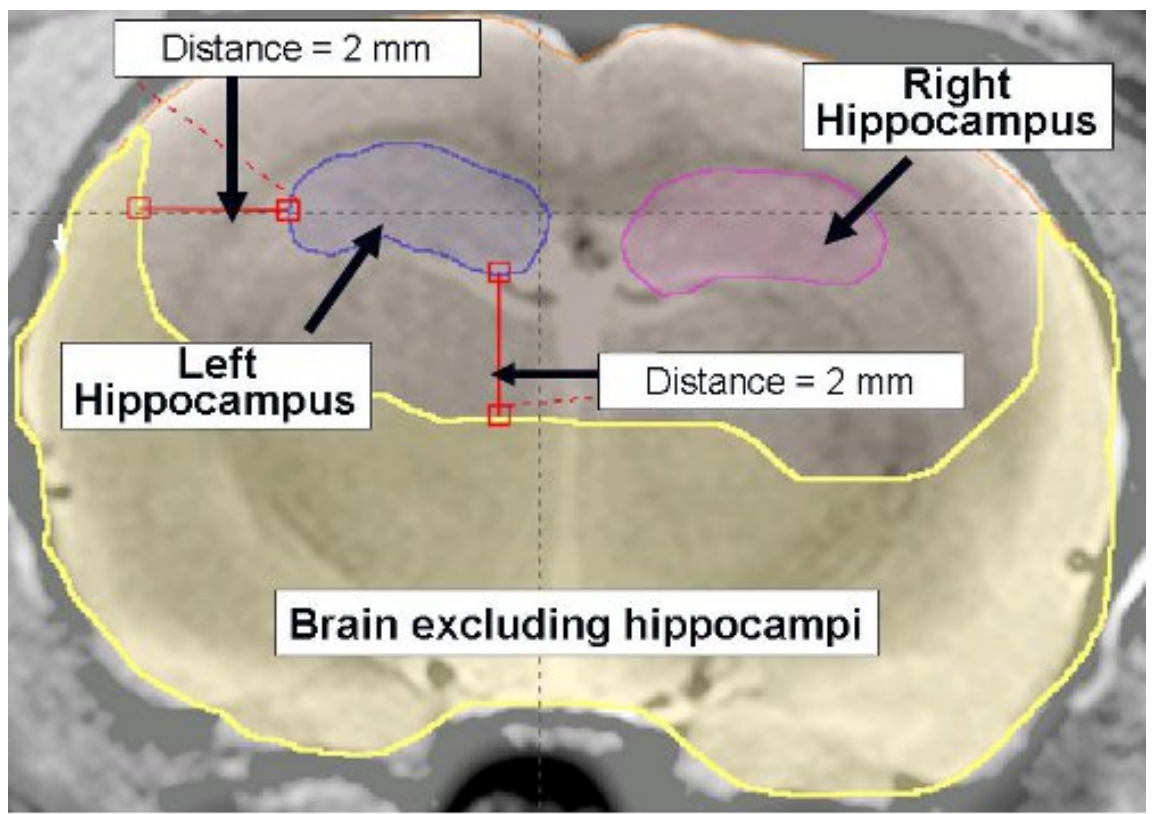

Figure 3. Contoured hippocampal regions of the brain. Subsequent to image fusion, hippocampi and brain excluding hippocampi are identified and contoured axially defining specific volumetric regions for these organs. These regions provide anatomical and volume information necessary for adjusting the dose distribution to the desired target(s). 


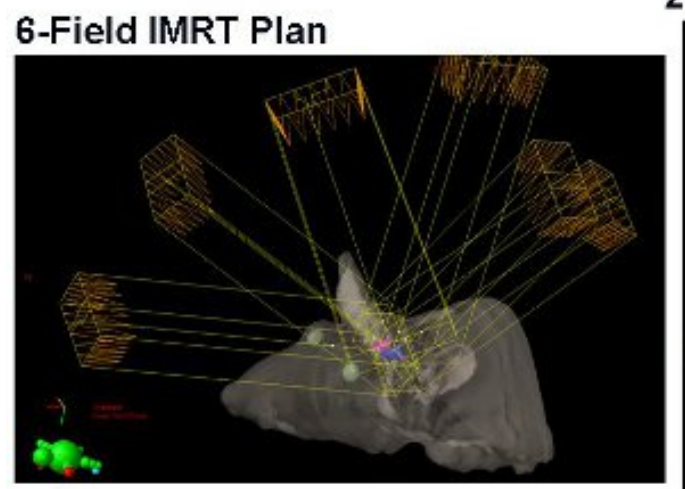

\section{2-Arc RapidArc Plan}

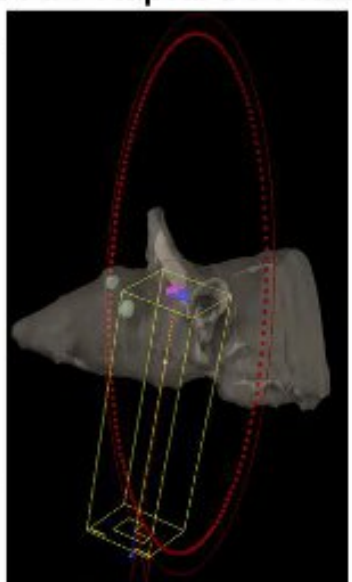

Figure 4. High precision irradiation options using intensity-modulated radiation therapy (IMRT) or volumetrically-modulated arc therapy (VMAT) in the form of RapidArc. These techniques deliver $6 \mathrm{MV}$ photon beams either as multiple static trajectories that can converge on very small target volumes (IMRT), or dynamically as one or more arcs focused on the target region (RapidArc).

\section{Single Hippocampus Irradiation}

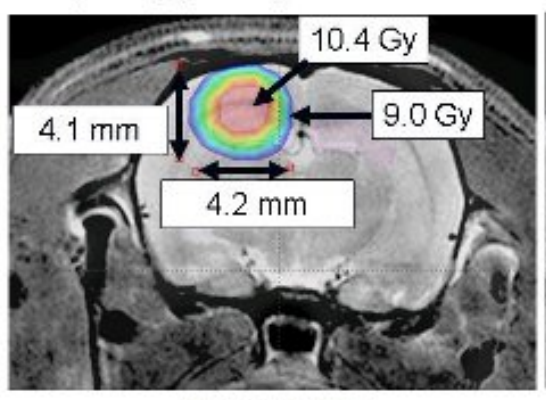

6-Field IMRT

\section{Both Hippocampi Irradiation}

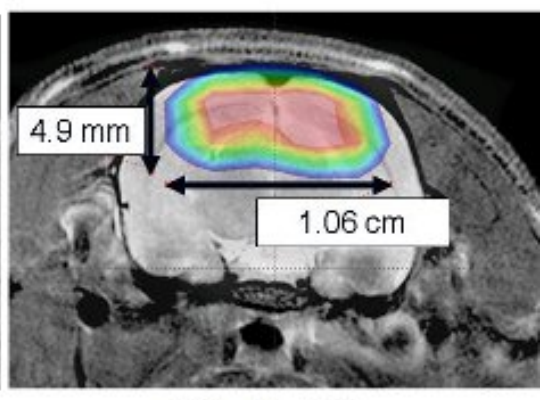

\section{2-Arc RapidArc}

Figure 5. ECLIPSE calculated doses superimposed to the axial images. Doses shown are for single or dual hippocampi irradiation treatment plans.

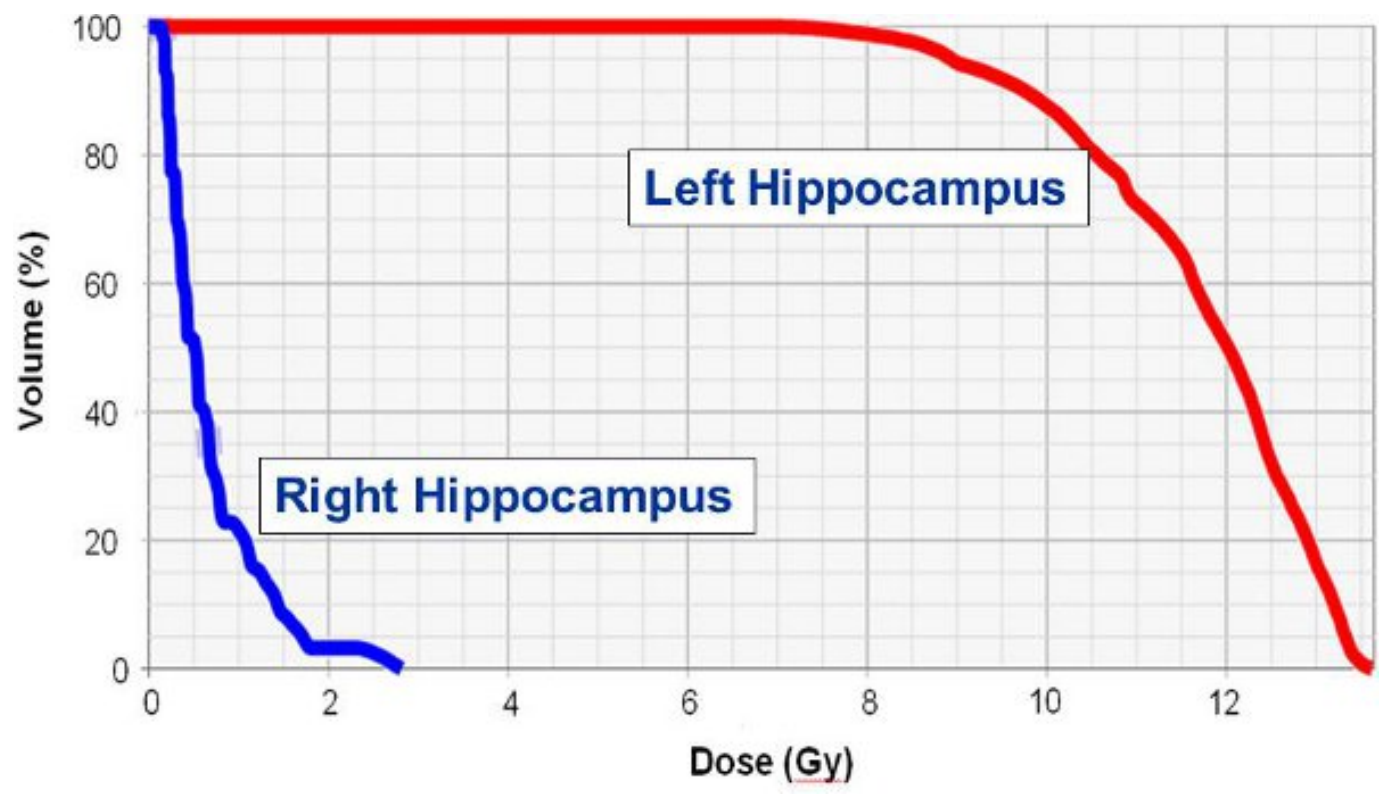

Figure 6. ECLIPSE calculated dose-volume histogram. Data contrasts the percentage of the irradiated versus the non-irradiated hippocampal volume under the single hippocampus treatment plan shown in Fig. 5. 


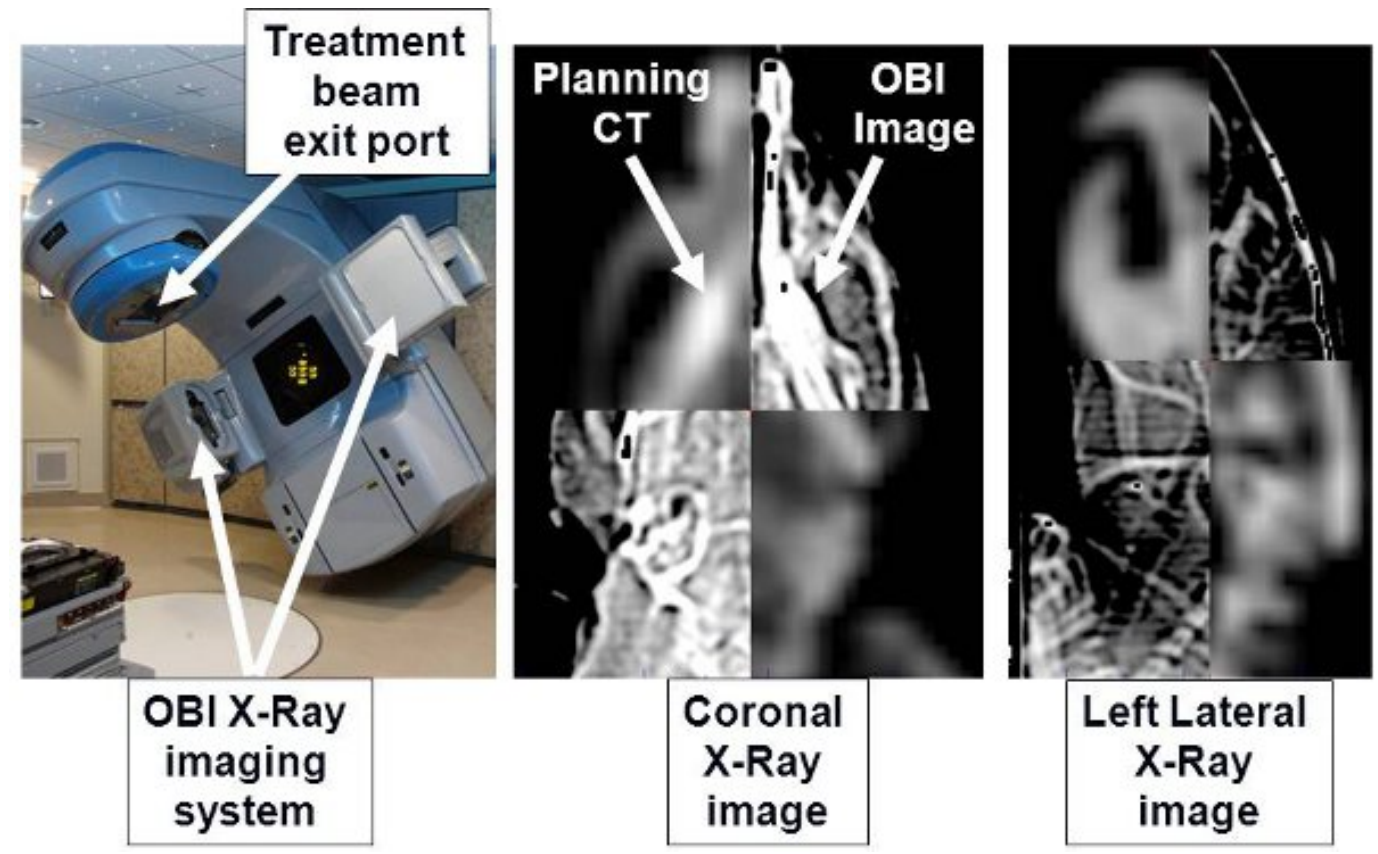

Figure 7. Image guided rat positioning for radiotherapy. Orthogonal digitally-reconstructed radiograph (DRR) images generated from the CT treatment planning data in ECLIPSE are fused to orthogonal x-ray images of the rat on the treatment table taken with the Trilogy's on-board imaging (OBI) system. The DRRs are weighted on bone density that highlights the skull and other bony landmarks which facilitate co-registration with the $\mathrm{OBI}$ images. Matching of these sets provide treatment table position shifts that must be made to achieve the co-registration of the $\mathrm{CT}$ and $\mathrm{x}$-ray images. 


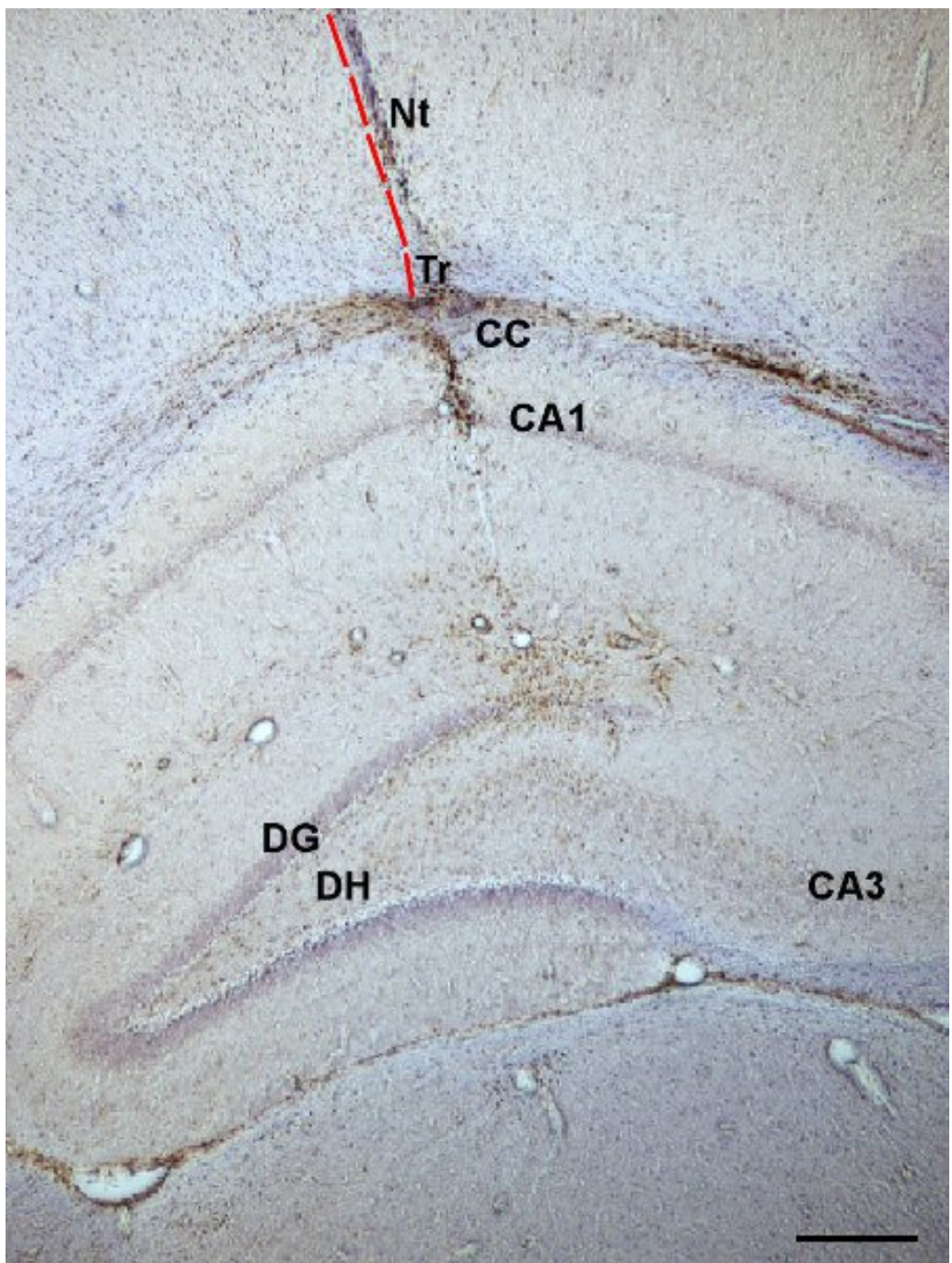

Figure 8. Location of transplanted NSCs following stereotaxic surgery. At 1-month post-transplantation, animals were perfused, brains were sectioned and stained with BrdU (to detect transplanted NSCs) and counter stained with hematoxylin. The needle track (Nt, red line), indicates the injection trajectory that deposited NSCs at the transplant-release site (Tr), just below the corpus callosum (CC) and above the CA1. Transplanted NSCs showed extensive migration from Tr throughout the host hippocampus (dentate gyrus, DG; dentate hilus, DH; CA1 and CA3 subfields; magnification $\times 4$ ). Scale bar, $200 \mu \mathrm{m}$.

\section{Discussion}

Considerable research is underway exploring the myriad of ways that stem cells can be used clinically to restore normal functions to damaged, aged and diseased tissues ${ }^{8}$. The eventual realization of these efforts will require a detailed understanding of the behavior of engrafted cells within unique microenvironments that are distinct from undamaged normal tissue. Our work has demonstrated that within the irradiated tissue bed, cranially engrafted NSCs can functionally restore cognition, where they survive, migrate and differentiate along neural and glial lineages ${ }^{2}$. Precisely how these cells mediate recovery of cognition is uncertain at present, but does depend on conducting a series of carefully controlled experimental procedures in a reproducible manner. We have detailed these critical procedures here in efforts to expedite the translational potential of stem cell therapies for ameliorating adverse cognitive effects associated with the clinical management of brain and other forms of cancer. Additional considerations that are likely to have a significant impact of the quality of data are highlighted below.

Transplantation based therapies depend on stem cells as the critical reagent, and accordingly, care must be exercised to properly characterize cultures, maintain sterility and use matched passage numbers for reliability of results. Transplanted human stem cells were labeled with BrdU prior to surgery, to provide one means for tracking them in vivo. Under our experimental conditions, transplanted NSCs did not undergo extensive proliferation, so that dilution of the BrdU label was not problematic. Alternatively, transplanted human stem cells can be distinguished from host cells by immunostaining for human specific markers such as nuclear matrix protein (h-NUC or hNUMA) ${ }^{9}$ or human-specific nuclear antigen $(\mathrm{HuNu})^{2}$. Human stem cells could also be labeled with a variety of fluorescent markers to facilitate their identification within the host brain.

Attention to irradiation parameters define precise dose delivery techniques, and those described here model current clinical practices in radiation oncology. Reproducible transplantation of stem cells in the brain is also critical, and is accomplished using a digital stereotaxic instrument. The capability to precisely micromanipulate a micro-syringe for stem cell implantation in small brain structures like the hippocampus greatly reduces human error. With this apparatus, NSCs were transplanted in four distinct sites spanning the anterior to posterior regions of the rat hippocampus. 
Dorso-ventral (DV) coordinates were determined based on experience with athymic nude (ATN) rats such that surgical procedures would not cause damage to the hippocampal formation ${ }^{2}$. A coronal section through the rat brain reveals key structures of the hippocampal formation including the dentate gyrus (DG), the dentate hilus (DH) and CA1 and CA3 subfields (Fig. 8). Transplanted NSCs, introduced dorsally from the visible needle tract (Nt, redline), are visualized as darkly staining (brown) cells deposited at the transplant-release site (Tr) just below the corpus callosum (CC), that subsequently migrate throughout the septo-temporal axis of the hippocampus.

\section{Disclosures}

No conflicts of interest declared.

\section{Acknowledgements}

This work was supported by NIH NINDS grant R01 NS074388 581 (C.L. Limoli), California Institute for Regenerative Medicine (CIRM) Grant RS1-00413 (C.L.L.), CIRM Training Grant TG2- 0115 (M.M.A.), and a CIRM Grant to JOVE in support of the video documentation.

\section{References}

1. Acharya, M.M., et al. Consequences of ionizing radiation-induced damage in human neural stem cells. Free. Radic. Biol. Med., doi:S0891-5849(10)00504-6 [pii] 10.1016/j.freeradbiomed.2010.08.021 (2010).

2. Acharya, M.M., et al. Rescue of radiation-induced cognitive impairment through cranial transplantation of human embryonic stem cells. Proc. Natl. Acad. Sci. U. S. A. 106, 19150-19155, doi:0909293106 [pii] 10.1073/pnas.0909293106 (2009).

3. Boyer, A. in Treatment planning in Radiation Oncology (ed. FM Khan) Ch. 11, (Lippincott Williams \& Wilkins 2007).

4. Lawson, J.D., et al. Intracranial application of IMRT based radiosurgery to treat multiple or large irregular lesions and verification of infra-red frameless localization system. J. Neurooncol. 97, 59-66, doi:10.1007/s11060-009-9987-0 (2010).

5. Ling, C.C., et al. Commissioning and quality assurance of RapidArc radiotherapy delivery system. Int. J. Radiat. Oncol. Biol. Phys. 72, 575-581, doi:S0360-3016(08)02464-4 [pii] 10.1016/j.ijrobp.2008.05.060 (2008).

6. Otto, K. Volumetric modulated arc therapy: IMRT in a single gantry arc. Med. Phys. 35, 310-317 (2008).

7. Paxinos, G. \&, Watson, C. The Rat Brain in stereotaxic coordinates. 6th edn, (Elsevier, 2007).

8. Hess, D.C. \& Borlongan, C.V. Cell-based therapy in ischemic stroke. Expert. Rev. Neurother. 8, 1193-1201, doi:10.1586/14737175.8.8.1193 (2008).

9. Hefferan, M.P., Johe, K., Feldman, E.L., Lunn, J.S., \&, Marsala, M. Optimization of immunosuppressive therapy for spinal grafting of human spinal stem cells in a rat model of ALS. Cell. Transplant., doi:ct2097hefferan [pii] 10.3727/096368910X564553 (2011). 